\title{
Trade-off relations on CHSH tests for multi-qubit pure states
}

\author{
Meiyu Cui ${ }^{1,2}$, Xiaofen Huang ${ }^{1}$, Tinggui Zhang ${ }^{1, \dagger}$ \\ 1 School of Mathematics and Statistics, Hainan Normal University, \\ Haikou 571158, P. R. China \\ 2 Center School of Anji Vocational Education, Huzhou, 313301, P. R. China \\ $\dagger$ Correspondence to tinggui333@163.com
}

\begin{abstract}
We study the trade-off relations on the maximal violation of CHSH tests for the multi-qubit pure states. Firstly, according to the classification of 3-qubit pure states under stochastic local operations and classical communication, four trade-off relations on CHSH tests are provided. The process of proof also provides the method of calculating its exact value. Then we consider the multi-qubit quantum systems, and we prove that the conjecture in [PRA 94, 042105 (2016)] is right for some special 4-qubit or more qubit pure states.
\end{abstract}

PACS numbers: 03.67.-a, 02.20.Hj, 03.65.-w 


\section{INTRODUCTION}

Quantum entanglement as one of the most fascinating features of quantum mechanics has been investigated for decades. The relation of quantum entanglement and the violation of Bell's inequalities [1 3] is one of the key issues in quantum information. And the existence of Bell inequalities [5] and their observed violation in experiments have had a very deep impact on the way we look at quantum mechanics. Quantum mechanics exhibits the nonlocality of nature by violation of the Bell inequality.

Entanglement and nonlocality both are essential resource in quantum information theory, and establishing the link between them is intriguing. Quantum entanglement is considered to be the most nonclassical manifestation of quantum mechanics. It is exciting to know which states are both entangled and nonlocal. Quantum entanglement coincides with the violation of Bell inequalities for pure quantum states. Any pure entangled state violate a Bell inequality, and all bipartite pure entangled state violate the Bell inequality and the magnitude of the violation is directly proportional to the amount entanglement of the states [6-12]. The necessary and sufficient condition for a 2-qubit mixed state to violate the Bell$\mathrm{CHSH}($ Clauser-Horrne-Shimony-Holt) inequality has been derived [13]. A key property of entanglement is that a quantum system entangled with one other limits its entanglement with the remaining ones. This phenomenon is known as quantum monogamy and has recently been widely studied [14 17]. In particular, the authors of [16] presented the analytical trade-off relations obeyed by the CHSH test of pairwise qubits in a 3-qubit system.

Two states have the same kind of entanglement if they can be obtained with certainty from each other via local operation and classical communication (LOCC) with nonzero probability. In [18], W.Dür et al. presented that SLOCC splits the set of pure states of 3-qubit into six inequivalent classes.

\section{Class A-B-C(product states)}

$$
\left|\psi_{A-B-C}\right\rangle=|0\rangle|0\rangle|0\rangle
$$

where the rank of the reduced density matrices of $\left|\psi_{A-B-C}\right\rangle$ satisfies $r\left(\rho_{A}\right)=r\left(\rho_{B}\right)=$ $r\left(\rho_{C}\right)=1$.

\section{Class A-BC,AB-C and C-AB(bipartite entanglement states)}

$$
\left|\psi_{A-B C}\right\rangle=|0\rangle\left(C_{\delta}|0\rangle|0\rangle+S_{\delta}|1\rangle|1\rangle\right),
$$


where we denote $\operatorname{Sin} \delta, \operatorname{Cos} \delta$ with $S_{\delta}, C_{\delta}$ respectively in abbreviation in this paper, and $C_{\delta} \geq S_{\delta}>0, r\left(\rho_{A}\right)=1, r\left(\rho_{B}\right)=r\left(\rho_{C}\right)=2$, and similarly for $\left|\psi_{B-A C}\right\rangle$ and $\left|\psi_{C-A B}\right\rangle$.

\section{W-class}

$$
\left|\psi_{W}\right\rangle=\sqrt{a}|001\rangle+\sqrt{b}|010\rangle+\sqrt{c}|100\rangle+\sqrt{d}|000\rangle
$$

where $a, b, c>0, d=1-(a+b+c) \geq 0$.

\section{GHZ-class}

$$
\left|\psi_{G H Z}\right\rangle=\sqrt{k}\left(C_{\delta}|0\rangle|0\rangle|0\rangle+S_{\delta} e^{i \varphi}\left|\psi_{A}\right\rangle\left|\psi_{B}\right\rangle\left|\psi_{C}\right\rangle\right)
$$

where $\left|\psi_{A}\right\rangle=C_{\alpha}|0\rangle+S_{\alpha}|1\rangle,\left|\psi_{B}\right\rangle=C_{\beta}|0\rangle+S_{\beta}|1\rangle,\left|\psi_{C}\right\rangle=C_{\gamma}|0\rangle+S_{\gamma}|1\rangle$, and $\kappa=(1+$ $\left.2 C_{\delta} S_{\delta} C_{\alpha} C_{\beta} C_{\gamma} C_{\varphi}\right)^{-1} \in\left(\frac{1}{2},+\infty\right)$ is a normalization factor, the ranges of five parameters are $\delta \in\left(0, \frac{\pi}{4}\right], \alpha, \beta, \gamma \in\left(0, \frac{\pi}{2}\right]$ and $\varphi \in(0,2 \pi]$.

Now we consider the 3-qubit quantum states via the violation of Bell inequality by the two-part reduced density matrix of the 3-qubit quantum states.

For a 2-qubit state $\rho$, it can be expressed according to the Bloch representation, that is

$$
\rho=\frac{1}{4}\left(I \otimes I+\sum_{i=1}^{3} r_{i} \sigma_{i} \otimes I+\sum_{j=1}^{3} s_{j} I \otimes \sigma_{j}+\sum_{i, j=1}^{3} m_{i j} \sigma_{i} \otimes \sigma_{j}\right),
$$

where $I$ is the identity matrix, $\sigma_{i}, i=1,2,3$ are the Pauli matrices, and the coefficients $r_{i}=\operatorname{tr}\left(\rho \sigma_{i} \otimes I\right), s_{j}=\operatorname{tr}\left(\rho I \otimes \sigma_{j}\right)$, the correlation matrix $m_{i j}=\operatorname{tr}\left(\rho \sigma_{i} \otimes \sigma_{j}\right)$, and $M=\left(m_{k l}\right)$ is a matrix with size $3 \times 3$.

The famous CHSH inequality was derived in 1969 [5]

$$
|\langle C H S H\rangle| \leq 2
$$

where CHSH operator is $A_{1} B_{1}+A_{1} B_{2}+A_{2} B_{1}-A_{2} B_{2}, A_{1}, A_{2}$ are observables on Alice, $B_{1}, B_{2}$ are observables on Bob. Combining the Bloch representation, one has the $\mathrm{CHSH}$ relation for bipartite quantum state $\rho$ given in [19]

$$
\langle C H S H\rangle_{\rho}=2 \sqrt{\tau_{1}+\tau_{2}}
$$

where $\tau_{1}$ and $\tau_{2}$ are the two largest eigenvalues of the matrix $M^{\dagger} M$.

Later Qin et al. presented a trade-off relation about CHSH inequality for the 3-qubit states in [16]. For any 3-qubit state $\rho_{A B C}$ in quantum system $\mathbf{H}^{\mathbf{A}} \otimes \mathbf{H}^{\mathbf{B}} \otimes \mathbf{H}^{\mathbf{C}}$, the maximal violation of $\mathrm{CHSH}$ tests on pairwise bipartite states satisfies the following trade-off relation:

$$
\langle C H S H\rangle_{\rho_{A B}}^{2}+\langle C H S H\rangle_{\rho_{B C}}^{2}+\langle C H S H\rangle_{\rho_{A C}}^{2} \leq 12
$$


where $\rho_{A B}=\operatorname{tr}_{C} \rho_{A B C}, \rho_{A C}=\operatorname{tr}_{B} \rho_{A B C}, \rho_{B C}=\operatorname{tr}_{A} \rho_{A B C}$.

In this paper we derive four CHSH trade-off relations based on the six classes of 3-qubit quantum pure states.

\section{SPECIFIC TRADE-OFF RELATIONS FOR 3-QUBIT PURE STATES}

According to the six classes of 3-qubit pure stats, we can obtain different trade-off relations respectively.

Theorem 1 For the 3-qubit product states(class $A-B-C$ ), the maximal violation of $C H S H$ inequality tests on pairwise bipartite states satisfies the following trade-off relation

$$
\langle C H S H\rangle_{\rho_{A B}}^{2}+\langle C H S H\rangle_{\rho_{B C}}^{2}+\langle C H S H\rangle_{\rho_{A C}}^{2}=12 .
$$

Proof: Let the 3-qubit product pure state be $|\psi\rangle=|0\rangle|0\rangle|0\rangle$, which has density matrix $\rho_{A B C}=|\psi\rangle\langle\psi|=| 000\rangle\langle 000|$.

So the reduced density matrices are $\rho_{B C}=\rho_{A C}=\rho_{A B}=|00\rangle\langle 00|$, and they are in the 2-qubit quantum subsystem.

Now we decomposite the reduced density matrix $\rho_{A B}$ in Bloch representation, and we can get the entries of correlation matrix are

$$
\begin{gathered}
m_{11}^{A B}=m_{12}^{A B}=m_{13}^{A B}=m_{21}^{A B}=m_{22}^{A B}=m_{23}^{A B}=m_{31}^{A B}=m_{32}^{A B}=0, \\
m_{33}^{A B}=1 .
\end{gathered}
$$

So the correlation matrix $M^{A B}$ is

$$
M^{A B}=\left(\begin{array}{lll}
0 & 0 & 0 \\
0 & 0 & 0 \\
0 & 0 & 1
\end{array}\right),
$$

and the eigenvalues of matrix $M^{A B}{ }^{\dagger} M^{A B}$ are $\tau_{1}=1$ and $\tau_{2}=\tau_{3}=0$.

Then one get the mean value of CHSH operator for the reduced matrices $\rho_{A B}, \rho_{B C}, \rho_{A C}$

$$
\langle C H S H\rangle_{\rho_{A B}}^{2}=\langle C H S H\rangle_{\rho_{B C}}^{2}=\langle C H S H\rangle_{\rho_{A C}}^{2}=4\left(\tau_{1}+\tau_{3}\right)=4 .
$$

Thus we can obtain the trade-off CHSH relation (8) for the 3-qubit product states(class A-B-C) in the theorem. 
Theorem 2 For bipartite entanglement states(class $A-B C, A B-C, C-A B) \rho_{A B C}$, it satisfies the following trade-off relation

$$
8 \leq\langle C H S H\rangle_{\rho_{A B}}^{2}+\langle C H S H\rangle_{\rho_{B C}}^{2}+\langle C H S H\rangle_{\rho_{A C}}^{2}<12
$$

Proof: Without loss of generality, let the bipartite entanglement states (class A-BC) be $\left|\psi_{A-B C}\right\rangle=|0\rangle\left(C_{\delta}|0\rangle|0\rangle+S_{\delta}|1\rangle|1\rangle\right)$, and it is with density matrix $\rho_{A B C}$,

$$
\rho_{A B C}=C_{\delta}^{2}|000\rangle\left\langle 000\left|+S_{\delta} C_{\delta}\right| 011\right\rangle\left\langle 000\left|+C_{\delta} S_{\delta}\right| 000\right\rangle\left\langle 011\left|+S_{\delta}^{2}\right| 011\right\rangle\langle 011|,
$$

where $\quad C_{\delta} \geq S_{\delta}>0$, and the ranks of reduced matrices satidfy $r\left(\rho_{A}\right)=1, r\left(\rho_{B}\right)=r\left(\rho_{C}\right)=$ 2 .

Taking trace on the subsystem $\mathbf{H}^{\mathrm{C}}$, one has the reduced matrix $\rho_{A B}$ like this

$$
\rho_{A B}=C_{\delta}^{2}|00\rangle\left\langle 00\left|+S_{\delta}^{2}\right| 01\right\rangle\langle 01|
$$

and we rewrite $\rho_{A B}$ with matrix form

$$
\rho_{A B}=\left(\begin{array}{cccc}
C_{\delta}^{2} & 0 & 0 & 0 \\
0 & S_{\delta}^{2} & 0 & 0 \\
0 & 0 & 0 & 0 \\
0 & 0 & 0 & 0
\end{array}\right)
$$

Then denoting $\rho_{A B}$ in Bloch representation, we can get the correlation matrix $M^{A B}$ of $\rho_{A B}$

$$
M^{A B}=\left(\begin{array}{ccc}
0 & 0 & 0 \\
0 & 0 & 0 \\
0 & 0 & C_{\delta}^{2}-S_{\delta}^{2}
\end{array}\right) .
$$

Since the matrix $\left(M^{A B}\right)^{\dagger} M^{A B}$ has the eigenvalues $\tau_{1}=\left[C_{\delta}^{2}-S_{\delta}^{2}\right]^{2}$ and $\tau_{2}=\tau_{3}=0$, we arrive at that

$$
\langle C H S H\rangle_{\rho_{A B}}^{2}=4\left(\tau_{1}+\tau_{3}\right)=4\left(C_{\delta}^{2}-S_{\delta}^{2}\right)^{2}
$$

Similarly we get equalities about CHSH equalities of reduced matrices $\rho_{B C}$ and $\rho_{A C}$

$$
\begin{aligned}
& \langle C H S H\rangle_{\rho_{B C}}^{2}=16 C_{\delta}^{2} S_{\delta}^{2}+4 \\
& \langle C H S H\rangle_{\rho_{A C}}^{2}=4\left(C_{\delta}^{2}-S_{\delta}^{2}\right)^{2} .
\end{aligned}
$$


Combining equalities (10) and (11), we have

$$
\begin{aligned}
& \langle C H S H\rangle_{\rho_{A B}}^{2}+\langle C H S H\rangle_{\rho_{B C}}^{2}+\langle C H S H\rangle_{\rho_{A C}}^{2} \\
= & 8\left(C_{\delta}^{2}-S_{\delta}^{2}\right)^{2}+16 C_{\delta}^{2} S_{\delta}^{2}+4=8 C_{2 \delta}^{2}+4 S_{2 \delta}^{2}+4=4 C_{2 \delta}^{2}+8 .
\end{aligned}
$$

Because of $C_{\delta}>0$ and $C_{2 \delta}=1-2 C_{\delta}^{2}<1$, thus $0 \leq C_{2 \delta}^{2}<1$. Also due to the range of $C_{2 \delta}^{2}$ and Eq.(12), then we can get that the trade-off relation (9).

The following conclusion is about the W-class state.

Theorem 3 For the $W$-class state $\rho_{A B C}$, there is the following trade-off relation

$$
8<\langle C H S H\rangle_{\rho_{A B}}^{2}+\langle C H S H\rangle_{\rho_{B C}}^{2}+\langle C H S H\rangle_{\rho_{A C}}^{2}<12
$$

Proof: Let the 3-qubit pure state of W-class be $\left|\psi_{W}\right\rangle=\sqrt{a}|001\rangle+\sqrt{b}|010\rangle+\sqrt{c}|100\rangle+$ $\sqrt{d}|000\rangle$, where $a, b, c>0, d=1-(a+b+c) \geq 0$.

By calculating the eigenvalues of correlation matrix of $\left|\psi_{W}\right\rangle$ and borrowing the results of Ref.[20], we can obtain the CHSH relations in the flowing

$$
\begin{gathered}
\langle C H S H\rangle_{\rho_{A B}}^{2}=2[1+12 a b-4 a c-4 b c+\sqrt{V}], \\
\langle C H S H\rangle_{\rho_{A C}}^{2}=2[1+12 a c-4 a b-4 b c+\sqrt{V}], \\
\langle C H S H\rangle_{\rho_{B C}}^{2}=2[1+12 b c-4 a b-4 a c+\sqrt{V}],
\end{gathered}
$$

where $V=\left[(\sqrt{a}+\sqrt{b}+\sqrt{c})^{2}+d\right]\left[(\sqrt{a}+\sqrt{b}-\sqrt{c})^{2}+d\right]\left[(\sqrt{a}-\sqrt{b}+\sqrt{c})^{2}+d\right][(-\sqrt{a}+\sqrt{b}+$ $\left.\sqrt{c})^{2}+d\right]$. Therefore

$$
\begin{aligned}
& \langle C H S H\rangle_{\rho_{A B}}^{2}+\langle C H S H\rangle_{\rho_{B C}}^{2}+\langle C H S H\rangle_{\rho_{A C}}^{2} \\
= & 2[3+4 a b+4 a c+4 b c+3 \sqrt{V}]=2[3(1+\sqrt{V})+4(a b+a c+b c)] .
\end{aligned}
$$

We consider the above formula as a function of parameters $a, b$ and $c$, then it's a continuous function of parameters $a, b$ and $c$. Thus, when $a, b$ and $c$ all tend to zero, the value of this function approaches 12 . When $a=b$ approaches $1 / 2$, the value of the function approaches 8. In fact, for any 3-qubit pure state, the value of the function will be between 8 and 12 . The upper bound has been proved [16], and the lower bound is obvious. Because the sum of all eigenvalues of $M_{A B}^{\dagger} M_{A B}, M_{A C}^{\dagger} M_{A C}$ and $M_{B C}^{\dagger} M_{B C}$ is 3 , and here we use the largest two eigenvalues of each matrix, so this value will not be less than $3 \times 2 / 3=2$, that is $\langle C H S H\rangle_{\rho_{A B}}^{2}+\langle C H S H\rangle_{\rho_{B C}}^{2}+\langle C H S H\rangle_{\rho_{A C}}^{2} \geq 4 \times 2=8$. Therefore, we get the trade-off relation (13). 
Theorem 4 For the GHZ-class state $\rho_{A B C}$, there is the following trade-off relation

$$
8 \leq\langle C H S H\rangle_{\rho_{A B}}^{2}+\langle C H S H\rangle_{\rho_{B C}}^{2}+\langle C H S H\rangle_{\rho_{A C}}^{2} \leq 12
$$

Proof: Let the GHZ-class pure state be with form

$$
\left|\psi_{G H Z}\right\rangle=\sqrt{k}\left(C_{\delta}|0\rangle|0\rangle|0\rangle+S_{\delta} e^{i \varphi}\left|\psi_{A}\right\rangle\left|\psi_{B}\right\rangle\left|\psi_{C}\right\rangle\right)
$$

where $\left|\psi_{A}\right\rangle=C_{\alpha}|0\rangle+S_{\alpha}|1\rangle,\left|\psi_{B}\right\rangle=C_{\beta}|0\rangle+S_{\beta}|1\rangle,\left|\psi_{C}\right\rangle=C_{\gamma}|0\rangle+S_{\gamma}|1\rangle$, and $\kappa=(1+$ $\left.2 C_{\delta} S_{\delta} C_{\alpha} C_{\beta} C_{\gamma} C_{\varphi}\right)^{-1} \in\left(\frac{1}{2},+\infty\right)$ is a normalization factor, the ranges of five parameters are $\delta \in\left(0, \frac{\pi}{4}\right], \alpha, \beta, \gamma \in\left(0, \frac{\pi}{2}\right]$ and $\varphi \in(0,2 \pi]$.

Via computing the eigenvalues of reduced density matrix of $\left|\psi_{G H Z}\right\rangle$, and we can get CHSH relations in the flowing

$$
\begin{aligned}
\langle C H S H\rangle_{\rho_{A B}}^{2} & =4 \times\left[1+\frac{\left(C_{\alpha}^{2}-C_{\beta}^{2}-C_{\gamma}^{2}+2 C_{\beta}^{2} C_{\gamma}^{2}\right) S_{2 \delta}^{2}-C_{\alpha}^{2} C_{\beta}^{2} C_{\gamma}^{2} S_{2 \delta}^{2}}{\left(1+C_{\alpha}^{2} C_{\beta}^{2} C_{\gamma}^{2} S_{2 \delta}^{2}\right)^{2}}\right], \\
\langle C H S H\rangle_{\rho_{A C}}^{2} & =4 \times\left[1+\frac{\left(C_{\beta}^{2}-C_{\alpha}^{2}-C_{\gamma}^{2}+2 C_{\alpha}^{2} C_{\gamma}^{2}\right) S_{2 \delta}^{2}-C_{\alpha}^{2} C_{\beta}^{2} C_{\gamma}^{2} S_{2 \delta}^{2}}{\left(1+C_{\alpha}^{2} C_{\beta}^{2} C_{\gamma}^{2} S_{2 \delta}^{2}\right)^{2}}\right], \\
\langle C H S H\rangle_{\rho_{B C}}^{2} & =4 \times\left[1+\frac{\left(C_{\gamma}^{2}-C_{\alpha}^{2}-C_{\beta}^{2}+2 C_{\alpha}^{2} C_{\beta}^{2}\right) S_{2 \delta}^{2}-C_{\alpha}^{2} C_{\beta}^{2} C_{\gamma}^{2} S_{2 \delta}^{2}}{\left(1+C_{\alpha}^{2} C_{\beta}^{2} C_{\gamma}^{2} S_{2 \delta}^{2}\right)^{2}}\right] .
\end{aligned}
$$

therefore

$$
\begin{aligned}
& \left.\langle C H S H\rangle_{\rho_{A B}}^{2}+\langle C H S H\rangle_{\rho_{A C}}^{2}+C H S H\right\rangle_{\rho_{B C}}^{2} \\
= & 12+4 \times \frac{\left(2 C_{\alpha}^{2} C_{\beta}^{2}+2 C_{\alpha}^{2} C_{\gamma}^{2}+2 C_{\beta}^{2} C_{\gamma}^{2}-C_{\alpha}^{2}-C_{\beta}^{2}-C_{\gamma}^{2}-3 C_{\alpha}^{2} C_{\beta}^{2} C_{\gamma}^{2}\right) S_{2 \delta}^{2}}{\left(1+C_{\alpha}^{2} C_{\beta}^{2} C_{\gamma}^{2} S_{2 \delta}^{2}\right)^{2}} .
\end{aligned}
$$

Let $a=C_{\alpha}^{2}, b=C_{\beta}^{2}, c=C_{\gamma}^{2}$, where $a, b, c \in[0,1)$, we consider a function about variables $a, b, c$

$$
\begin{aligned}
f(a, b, c) & =2 C_{\alpha}^{2} C_{\beta}^{2}+2 C_{\alpha}^{2} C_{\gamma}^{2}+2 C_{\beta}^{2} C_{\gamma}^{2}-C_{\alpha}^{2}-C_{\beta}^{2}-C_{\gamma}^{2}-3 C_{\alpha}^{2} C_{\beta}^{2} C_{\gamma}^{2} \\
& =2 a b+2 a c+2 b c-a-b-c-3 a b c \\
& =a(b-1)+b(c-1)+c(a-1)+a b(1-c)+a c(1-b)+b c(1-a) \\
& =a(b-1)(1-c)+b(c-1)(1-a)+c(a-1)(1-b) \leq 0
\end{aligned}
$$

At the same time, we can get the lower bound of $f(a, b, c)$ is -1 , i. e.

$$
-1 \leq f(a, b, c) \leq 0
$$


and also $0<S_{2 \delta}^{2} \leq 1,\left(1+C_{\alpha}^{2} C_{\beta}^{2} C_{\gamma}^{2} S_{2 \delta}^{2}\right)^{2}>1$, thus we have

$$
0<\frac{S_{2 \delta}^{2}}{\left(1+C_{\alpha}^{2} C_{\beta}^{2} C_{\gamma}^{2} S_{2 \delta}^{2}\right)^{2}}<1 .
$$

Combining (17), (19), (20), we can obtain the trade-off relation (15).

Remark: In fact, if we only look at the conclusions of our theorems, we will find that after a lot of calculations we only get similar results. This makes our conclusions look a little weak. But we must emphasize that the really useful conclusions are the formulas $(10),(14)$ and (17) in the theorems proof, because they give the exact values of each specific quantum state.

A brief summary, for all six classes of the 3-qubit pure states, the quantity $\langle C H S H\rangle_{\rho_{A B}}^{2}+$ $\langle C H S H\rangle_{\rho_{B C}}^{2}+\langle C H S H\rangle_{\rho_{A C}}^{2}$ relative to $\mathrm{CHSH}$ operators been calculated accurately and bound between 8 and 12 .

\section{TRADE-OFF RELATIONS FOR MULTI-QUBIT PURE STATES}

Now, we study trade-off relations about CHSH for multi-qubit pure states. For all of 4-qubit pure states $\left|\psi_{A B C D}\right\rangle$, the authors of the paper 21] conjectured that the following inequality holds

$$
\operatorname{tr}\left[T_{A B} T_{A B}^{T}\right]+\operatorname{tr}\left[T_{A C} T_{A C}^{T}\right]+\operatorname{tr}\left[T_{A D} T_{A D}^{T}\right] \leq 3
$$

with respect to the bi-partitions $(\mathrm{AB}, \mathrm{CD}),(\mathrm{AC}, \mathrm{BD})$, and $(\mathrm{AD}, \mathrm{BC})$, and $T_{A B}=\operatorname{tr}\left[\rho_{A B C D} \sigma_{i} \otimes\right.$ $\left.\sigma_{j} \otimes I_{2} \otimes I_{2}\right], \rho_{A B C D}=\left|\psi_{A B C D}\right\rangle\left\langle\psi_{A B C D}\right|$ and so on. Through the discussion of the three-qubit pure state in the previous section, we found that the conjecture is correct in at least the following two situations.

(1) When $\left|\psi_{A B C D}\right\rangle$ is a fully separable state. Then by local unitary transformation $\left|\psi_{A B C D}\right\rangle=|0000\rangle$, therefore, $\operatorname{Tr}\left[T_{A B} T_{A B}^{T}\right]=\operatorname{Tr}\left[T_{A C} T_{A C}^{T}\right]=\operatorname{Tr}\left[T_{A D} T_{A D}^{T}\right]=1$, i.e $\operatorname{Tr}\left[T_{A B} T_{A B}^{T}\right]+\operatorname{Tr}\left[T_{A C} T_{A C}^{T}\right]+\operatorname{Tr}\left[T_{A D} T_{A D}^{T}\right]=3$.

(2) When $\left|\psi_{A B C D}\right\rangle$ is a generalized GHZ state. That is to say $\left|\psi_{A B C D}\right\rangle=\cos \theta|0000\rangle+$ $\sin \theta|1111\rangle$. It is easy to get the following facts $\operatorname{Tr}\left[T_{A B} T_{A B}^{T}\right]=\operatorname{Tr}\left[T_{A C} T_{A C}^{T}\right]=\operatorname{Tr}\left[T_{A D} T_{A D}^{T}\right]=$ $\cos \theta^{2}+\sin \theta^{2}=1$, i.e $\operatorname{Tr}\left[T_{A B} T_{A B}^{T}\right]+\operatorname{Tr}\left[T_{A C} T_{A C}^{T}\right]+\operatorname{Tr}\left[T_{A D} T_{A D}^{T}\right]=3$.

If like the three-qubit state, only the fully separable state and the GHZ state can give the upper bound for Eq.(7), then the conjecture of [21] is right. The conclusions of this kind of conjecture under the special case can be extended to more qubit cases. 


\section{CONCLUSIONS AND DISCUSSIONS}

In this paper, we have calculated in detail the restriction conditions of CHSH inequality violation values for different classes of three qubit quantum state. We have also studied the 4-qubit situation. These enables us to have a deeper understanding of monogamy relations of three and more qubit pure quantum states.

Acknowledgments This project is supported by the National Natural Science Foundation of China Grants No.11861031 and the Natural Science Foundation of Hainan province under Grants No.118QN230.

[1] A. Einstein, B. Podolsky and N. Rosen, Phys. Rev. 47, 777 (1935)

[2] E. SchrÖdinger, Naturwissenschaften 23, 807 (1935)

[3] J. S. Bell, Physics 1, 195 (1964)

[4] H. Cao, Z. Guo, Sci. China-Phys. Mech. Astron. 62(3), 030311 (2019)

[5] J. F. Clauser, M. A. Horne, A. Shimony, and R. A. Holt, Phys. Rev. Lett. 23, 880 (1969)

[6] N. Gisin, Phys. Lett. A 154, 201 (1991)

[7] N. Gisin and A. Peres, Phys. Lett. A 162, 15 (1992)

[8] S. Popescu and D. Rohrlich, Phys. Lett. A 166, 293 (1992)

[9] J. L. Chen, C. F. Wu, L. C. Kwek, and C. H. Oh, Phys. Rev. Lett. 93, 140407 (2004)

[10] M. Li and S. M. Fei, Phys. Rev. Lett. 104, 240502 (2010)

[11] S. X. Yu, Q. Chen, C. J. Zhang, C. H. Lai, and C. H. Oh, Phys. Rev. Lett. 109, 120402 (2012)

[12] S.H Jiang, Z.P. Xu, H.Y. Su, A. K. Pati, and J.L. Chen Phys. Rev. Lett. 120, 050403 (2018)

[13] R. Horodecki, P. Horodecki, and M. Horodecki, Phys. Lett. A. 200, 340 (1995)

[14] V.coffman, J.Kundu, and W.K.Wootters, Phys.Rev.A 61, 052306 (2000)

[15] N.Brunner, D.Cavalcanti, S.Pironio, V.Scarani and S.Wehner, Rev. Mod. Phys. 86, 419 (2014)

[16] H. H. Qin, S. M. Fei, and X. Q. Li, Phys. Rev. A. 92, 062339 (2015)

[17] S. Cheng and M.J.W.Hall, Phys. Rev. Lett. 118, 010401 (2017)

[18] W. Dür, G. Vidal and J. I. Cirac, Phys. Rev. A 62, 062314 (2000)

[19] M. Horodecki, P. Horodecki, and R. Horodecki, Phys. Lett. A. 210, 223 (1996)

[20] P. Pandya, A. Misra, I. Chakrabarty, Phys. Rev. A 94, 052126 (2016) 
[21] S. Cheng, A. Milne, M. J. W. Hall, and H. M. Wiseman, Phys. Rev. A 94, 042105 (2016) 